\title{
A novel approach for salvage treatment of non-small-cell lung cancer: percutaneous CT fluoroscopy-guided permanent seed brachytherapy for salvage treatment of lung cancer: long-term results of a case series
}

\author{
Stephen W. Doggett, MD, FACR', Shigeru Chino, MD², Todd Lempert, MD³ \\ IRadiation Oncology. Mission Regional Medical Center, Mission Viejo, CA, USA, ${ }^{2}$ Thoracic Surgery, Mission Regional Medical Center, Mission \\ Viejo, CA, USA, ${ }^{3}$ nterventional Radiology, Mission Regional Medical Center, Mission Viejo, CA, USA
}

\begin{abstract}
Purpose: We sought a curative technique to treat recurrent or primary non-small cell lung cancer (NSCLC) with a minimally invasive outpatient technique that could be repeated multiple times, had minimal pulmonary toxicity, and was readily available in the community setting. Percutaneous outpatient computed tomography (CT) fluoroscopy-guided permanent seed brachytherapy fits these criteria.

Material and methods: Eight lesions in five patients (three patients had two lesions each) with NSCLC were treated using outpatient percutaneous CT fluoroscopy-guided palladium-103 seed brachytherapy. At initial presentation, seven lesions had recurred following external beam radiation therapy (EBRT) and initial chemotherapy, and one lesion was treated with brachytherapy as a primary treatment.

Results: In five patients with eight lesions, there were no recurrences at follow-up from final implant, average follow-up of 58.1 months (range, 15-145 months). There were no acute complications requiring intervention and no longterm complications. One lesion required a second salvage implant 10 months after first implant. This lesion's original salvage $\mathrm{D}_{90}$ was but $90 \%$ and repeat implant achieved a $\mathrm{D}_{90}$ of $273 \%$. This salvaged site had not recurred at 43 -month follow-up after the second salvage implant.

Conclusions: Percutaneous CT fluoroscopy-guided permanent seed brachytherapy is a safe, efficacious, and cost effective primary and salvage treatment for lung cancer. CT-fluoroscopy resources are readily available in the community and are an effective alternative to stereotactic body radiation therapy (SBRT), intensity-modulated radiation therapy (IMRT)/ proton beam, radiofrequency ablation (RFA), and cryoablation (CA). Percutaneous CT fluoroscopy-guided permanent seed brachytherapy has an equivalent or better local control rate, a lower resource cost, and a far lower integral radiation dose than other therapies. We believe this is the first published article documenting the curative potential of percutaneous CT fluoroscopy-guided permanent seed brachytherapy for recurrent NSCLC with long-term follow-up. High $\mathrm{D}_{90}$ doses appear to be required to achieve complete response. Further studies are essential to confirm these findings.

Key words: lung cancer, brachytherapy, salvage, seeds, LDR.

\section{Purpose}

We sought a technique to treat recurrent or primary lung cancer with a minimally invasive outpatient technique that could be repeated multiple times, had minimal pulmonary toxicity, was readily available in the community setting, and had low resource cost. Percutaneous outpatient computed tomography (CT) fluorosco- py-guided permanent seed brachytherapy (CTGPSB) fit these criteria.

NSCLC is the foremost cause of worldwide cancer death, and the occurrence of NSCLC in 2013 in the USA was 182,400 cases [1,2]. Recurrent lung cancer following conventional radiations and chemotherapy is a vexing and common problem. Local control rates post-treatment are poor and range from $15 \%$ to $70 \%$ [3]. Nearly $70 \%$ of
Address for correspondence: Stephen Doggett, MD, 14642 Newport Ave \#470, Tustin CA, USA 92780, phone: +01 714573 9500, fax: +01 714573 9505, « e-mail: drdoggett@nocancer.com
Received: 02.06 .2018

Accepted: 13.03.2019

Published: 29.04.2019 
patients with lung cancer present with metastatic or locally advanced non-operable disease at the time of diagnosis and are candidates for treatment with primary radiations or chemo radiations [3,4]. Thirty to 85 percent of these locally advanced or metastatic patients will fail initial radiations or chemoradiations and are candidates for salvage therapy [3].

Various treatment modalities are currently used or have been used to promote local control of NSCLC. These treatments include increased radiation dose, salvage chemotherapies, stereotactic body radiation therapy (SBRT), proton beam, intensity-modulated radiation therapy (IMRT), radiofrequency ablation (RFA), and cryoablation [5].

One of the physicians from our group (SC) sought a technique to treat recurrent lung cancer with a minimally invasive outpatient technique that could be repeated multiple times, had minimal pulmonary toxicity, required no radiation shielding, was readily available in the community setting, and had a low financial and resource cost. CT-guided percutaneous implants utilizing low energy palladium-103 $\left({ }^{103} \mathrm{Pd}\right)$ seeds fit these criteria.

In 1991, our group was the first in the world to perform CTGPSB [6]. Our technique has been presented previously and has been described in detail $[6,7,8,9]$. Permanent seed brachytherapy utilizes low energy radioisotope sources $\left(22 \mathrm{KeV}^{103} \mathrm{Pd}\right.$, $35.5 \mathrm{Kev}$ iodine- $\left.125\left[{ }^{125} \mathrm{I}\right]\right)$ encased in a tiny titanium tube. Sources are $0.8 \mathrm{~mm}$ in diameter and $4.5 \mathrm{~mm}$ in length, and can be inserted through an 18-gauge needle. The low energy allows an extremely high deposition of radiations within the tumor over the decay life of the isotope, with a steep dose gradient fall-off immediately near the sources. This rapid fall-off of a dose as one moves farther from the source means little or no radiation delivered to tissues adjacent to the tumor. This is especially important in the situation where previous external beam radiation has been utilized, and in which additional radiations can increase the incidence of radiation pneumonitis and damage to the esophagus, tracheal bronchial tree, cardiovascular structures, and chest wall. We have not seen significant pulmonary function changes in our implanted patients, even patients undergoing as many as 16 implants to one lung over a 20 -month period, nor have we seen any lethal acute or delayed hemorrhage or radiation pneumonitis [8].

Body tissue shielding and physical inverse square law allows the patient to be discharged from the outpatient department, with no radiation precautions needed for patient, family, or healthcare providers [10]. The low energy palladium seeds can be utilized in any clinical setting without the need for concrete or steel shielding.

The introduction of CT-fluoroscopy technology into interventional radiology has permitted the seed implant procedure time to be reduced, and has improved lesion targeting and decreased the number of pleural punctures. CT-fluoroscopy involves acquisition of three contiguous CT slices of varying thickness, which allows the operator to take a volumetric approach with precise management of the needle tip. A high frame rate (up to eight images per second) and short delay $(0.3 \mathrm{sec})$ between needle movement as well as screen visualization of the needle movement provides near real-time motion picture style imaging.

Interventional radiologist (IR) skills are essential in the selection of the shortest path from skin puncture to lesion center to minimize needle transit length through normal lung tissue. We have noted a lower rate of asymptomatic pulmonary parenchymal hemorrhage with shorter needle transit lengths, and we have had no instances of significant perioperative hemoptysis or hemorrhage. We have used this technique to treat any site in the neck, thorax, pelvis, or abdomen, including trachea, peri-esophageal lesions, peri-aortic lesions, pulmonary apex, chest wall, and mediastinum. Subscapular lesions are accessible, with a frontal or lateral approach. Lesions abutting large vessels are accessible with the use of skillful interventional radiologists and vascular identification using intravenous contrast and CT fluoroscopic imaging.

\section{Material and methods}

This study was reviewed and approved by an ethical review board. The primary endpoint was cure rate of a small case series using ${ }^{103} \mathrm{Pd}$ seed implants for the treatment of non-small-cell lung cancer. The secondary endpoint was the occurrence of the Common Terminology Criteria Adverse Events (CTCAE) grades 2, 3, or 4. Eligibility criteria included those who had undergone the seed implants, subjects who had a recurrence from prior cancer therapy or require treatment of a primary cancer, have a minimum of one-year follow-up documented, and had undergone CT scan, chest X-ray, and clinical exam at the follow-up visit. Subjects who were excluded were those with no follow-up. Informed consent was not required by the ethical review board, since this was a minimal risk retrospective chart review. All patients had biopsy proven NSCLC.

Implants were carried out on an outpatient basis using the Siemens Somatom CT fluoroscopic scanner (Siemens Corporation, München, Germany), and patients were discharged the same day. Intravenous sedation was given using midazolam and fentanyl. Patients were continually monitored using continuous blood pressure monitors, electrocardiogram, and oxygen saturation by pulse oximetry. Each patient's position was based on proximity of the lesion to the chest wall and at interventional radiologists' discretion.

CT scanning was carried out and images reviewed by lead author and IR in the CT suite. IR placed a single 18-gauge $20-\mathrm{cm}$ in length prostate implant needle under local anesthesia into the designated mass under CT-fluoroscopic guidance. Lead author was at the couch side during needle placement. Lesions of $15 \mathrm{~mm}$ or less were generally treated with a single needle placement. IR placed the tip at the distal edge of the lesion. Tip position was again reviewed with lead author. Mick applicator was attached, and designated number of seeds were implanted along the needle track. Intermittent CT-fluoroscopic imaging during the case was used to confirm seed positioning. Multiple needle placements or repositioning of a needle after seed deposition was utilized for larger lesions. 
Pre-planning of implant had been carried out several days prior. Recent CT scan was imported into planning system (MIM Symphony ${ }^{\mathrm{TM}}$, Cleveland OH, USA). Isocenter was selected and best needle angle determined. Clinical target volume (CTV) was designated and 1-2 mm margins expanded to create planning target volume (PTV). Spinal cord doses, chest wall, and main stem bronchus doses were calculated as necessary. Standard planning template geometry was utilized to create an ideal implant. Planning was accomplished assuming needle parallelism with the understanding that intraoperative replication would be difficult. Implant was planned with ${ }^{103} \mathrm{Pd}$ (Theragenics Corporation, Buford, GA, USA), 2.0 to 4.0 U NIST/seed. Planned minimum dose to the CTV was 100 Gy. Maximal dose was disregarded due to desire for achieving high intra-tumoral dose, unless implant was adjacent to viscus, vessel, or chest wall.

During the implant, attempts were made to replicate the plan. Since increasing number of pleural punctures may increase the risk of bleeding and pneumothorax, we tried to minimize punctures; balancing the ideal needle and seed spacing against increasing numbers of pleural punctures. After needle(s) placement, lead author performed rapid "on the fly" cognitive treatment planning without computer support to confirm necessary seed numbers to reach planning dose of $100 \mathrm{~Gy}$. Mick applicator was then used to implant the seeds.

Typically, lesions of one to two $\mathrm{cm}$ in diameter were planned, with up to 6 needles, and implanted with one or two. Ideal implant technique would be one pleural puncture, then injection of seeds via Mick applicator, then withdrawal of the needle to just beneath the pleura, and redirection into another portion of the mass followed by further seed injections. Bony rib obstruction and impingement of the mass by bronchial and vascular structures were further challenges to satisfactory needle and seed positioning, and were overcome by increasing interventional radiologist experience.

Patients had the same day post-implant CT for pneumothorax surveillance and for post-implant dosimetry plan- ning at the completion of the implant. They were then taken to the recovery area and monitored for the next 6 hours and had a chest X-ray immediately prior to discharge.

Masses greater than $2 \mathrm{~cm}$ in diameter may require more than one needle puncture and several sub-pleural repositionings of each needle during seed deposition in order to ensure adequate lesion dosage. Intermittent CT fluoroscopy imaging is utilized during seed deposition to confirm appropriate seed spacing. CT fluoroscopy does not significantly increase radiation exposure to the patient. Exposure rates to personnel are not significantly different that from conventional CT procedures, although in the beam hand, the doses to the operator can be substantial [11]. This hand exposure is mitigated through the use of metal powder impregnated radiation attenuating sterile gloves.

We have performed over 600 CTGPSB procedures into the chest for multiple malignant cell types. Pneumothorax is uncommon and considered in our hands a minor complication usually requiring only pleural aspiration of the pneumothorax while the patient is still on the CT table. Upon CT confirmation of non-recurrence of the pneumothorax, a patient is sent to recovery and discharged home several hours later. Pleural vent placement is instituted if aspiration is not successful and the patient is discharged home with the appliance in place. The patient returns the next day for appliance removal. We have not had to abort a seeding procedure because of the appearance of pneumothorax or hemorrhage.

\section{Results}

We present eight lesions in five patients treated with CT fluoroscopy-guided percutaneous palladium seed implantation. Patient characteristics are listed in Table 1.

There were no serious adverse events in CTCAE (v4.0) grades two, three, or four. One subject experienced an asymptomatic pneumothorax that appeared intraoperatively and did not require intervention. Bleeding of $5 \mathrm{cc}$ or less occurred in each case and all patients were dis-

Table 1. Patient characteristics

\begin{tabular}{|c|c|c|c|c|c|c|c|c|c|c|c|}
\hline $\begin{array}{l}\text { Sub- } \\
\text { jects } \\
\text { no. }\end{array}$ & $\begin{array}{c}\text { Lesion } \\
\text { no. }\end{array}$ & $\begin{array}{c}\text { Gender } \\
\text { and age } \\
\text { (years) at } \\
\text { implant }\end{array}$ & $\begin{array}{l}\text { Stage at } \\
\text { implant }\end{array}$ & $\begin{array}{c}\mathrm{D}_{90} \\
\%\end{array}$ & $\begin{array}{c}\mathrm{V}_{100} \\
\%\end{array}$ & $\begin{array}{c}\mathrm{V}_{150} \\
\%\end{array}$ & Recurrence & $\begin{array}{l}\text { Follow-up } \\
\text { (months) }\end{array}$ & $\begin{array}{l}\text { Pneumo- } \\
\text { thorax }\end{array}$ & $\begin{array}{l}\text { Cell } \\
\text { type }\end{array}$ & Site \\
\hline 1 & 1 & M 73 & cT1c N1 cMO & 87.20 & $\mathrm{~N} / \mathrm{A}$ & N/A & No & 145 & No & $\mathrm{SCC}$ & Left hilum \\
\hline \multirow[t]{2}{*}{2} & 2 & F 58 & cT1bc NO cMO & 119.50 & 100 & 150 & No & 29 & No & Adeno & LLL \\
\hline & 3 & F 58 & cT1bc NO cMO & 30.30 & 100 & 150 & No & 29 & No & Adeno & LLL \\
\hline \multirow[t]{2}{*}{3} & 4 & F 67 & cT2b NO cMO & 193 & $\mathrm{~N} / \mathrm{A}$ & N/A & No & 53 & $\begin{array}{l}\text { Yes, } \\
\text { No Rx }\end{array}$ & SCC & RML \\
\hline & 5 & F 67 & cT1b NO cMO & $\begin{array}{c}273 \text { re- } \\
\text { implanted }\end{array}$ & N/A & N/A & $\begin{array}{c}\text { Yes re- } \\
\text { implanted }\end{array}$ & 43 & No & Adeno & LLL \\
\hline 4 & 6 & F 51 & rT1b NO cMO & 276 & N/A & N/A & No & 64 & No & Adeno & RML \\
\hline \multirow[t]{2}{*}{5} & 7 & M 63 & cT2b N3 cM0 & 349 & 100 & 100 & No & 73 & No & SCC & LUL \\
\hline & 8 & M 63 & cT2b N3 cM0 & 482 & 100 & 100 & No & 57 & No & SCC & Lingula \\
\hline
\end{tabular}

Rx-treatment, SCC - squamous cell carcinoma, Adeno - adenocarcinoma, LLL - left lower lobe, RML - right middle lobe, LUL - left upper lobe, N/A - not available 
charged home on the day of implant. Patients occasionally complained of transient mild chest wall pain attributed to needle insertion, which responded to conservative measures within a few days. There were no instances of radiation pneumonitis signs or symptoms, and no skin changes were noted after implantation.

The CTV was exactly what was treated; there were no margins in the CT volumes. $D_{90}$ varied from $30 \%$ to $482 \%$ (average, 226\%). The lowest outlier, 30\%, occurred due to this lesion being incidentally discovered during seeding of an adjacent lesion, and was implanted with residual seeds from the adjacent implanted lesion to avoid having patient return for a second procedure. This lesion has not recurred by imaging criteria. Treatment planning was performed from the scan at the completion of the procedure. Subjects 1,2 , and 5 underwent post-planning with MIM software. For subjects 3 and 4, post-planning was performed by the physicist with self-written software, and the charts were no longer retained due to the length of time that has gone by since their treatment, per institutional policy.

\section{Discussion}

An increasing number of patients is presented each year in the USA with recurrent lung cancer following standard chemoradiations. New targeted therapies show increasing promise in the management of these patients, but local control of the lesions remains a concern.

Various methods to improve local control rates have been considered such as increasing radiation dose. A metaanalysis of series that compared lung cancers treated with sequential chemotherapy and radiations, with those treated with simultaneous chemotherapy and radiations, suggested that improving local control had a beneficial effect on overall survival [12]. New salvage therapy strategies initially focused on improving local control by increasing the radiation dose delivered by conventional means and dose schedules, and by the addition of epidermal growth factor receptor (EGFR) inhibitors to systemic regimens. A recently reported phase three study (RTOG 0617) with 185 institutions included patients randomized to receive carboplatin plus either $60 \mathrm{~Gy}$ or $74 \mathrm{~Gy}$ in $2 \mathrm{~Gy}$ fractions with or without cetuximab. No benefit from increasing dose from 60 to 74 Gy was noted. There was no benefit from cetuximab, and the potential for harms (treatment-related deaths and severe esophagitis) from dose escalation and cetuximab was noted [13].

A second consideration is salvage chemotherapy. Salvage chemotherapies have had disappointing results. Second line chemotherapy after failed chemoradiations for lung cancer has shown limited efficacy $[14,15]$. EGFR and anaplastic lymphoma kinase (ALK) mutation analysis have led to altered strategies. Initial evaluation of tyrosine kinase inhibitors has shown some improvements in salvage therapy, although these improvements are typically transient $[16,17]$. EGFR inhibitors [Tarceva (erlotinib)] must be taken long-term and shows a 5-year survival in metastatic adenocarcinoma of $14.9 \%$ [18]. Checkpoint inhibitors [Opdivo (nivolumab)] have been recently introduced for second line treatment of squamous cell lung cancer and show promising results in early trials [19].
Because of the disappointing results of systemic molecules in salvaging recurrent lung cancer, attention has been directed to new local radiation therapies. CT-guided brachytherapy as described here, shows a high cure rate and insignificant toxicity.

CT fluoroscopy-guided permanent brachytherapy is limited only by the skill of the practitioner, and has a low cost and is less resource intensive than competing therapies. CT fluoroscopy does not significantly increase radiation exposure to the patient. Exposure rates to personnel are not significantly different that from conventional CT procedures, although in beam hand doses to the operator can be substantial [11].

Percutaneous CT fluoroscopy-guided permanent seed brachytherapy is a safe, effective, and cost effective primary and salvage treatment method for lung cancer and intrathoracic malignancies. Further studies are indicated to corroborate long-term survival and low toxicity of CTGPSB.

The ability to implant low energy sources directly into tumors results in a rapid fall-off of dose peripherally, with total sparing of adjacent normal tissues. A study comparing dosimetries of SBRT versus permanent seed brachytherapy for NSCLC showed extremely low-dose to normal tissue with brachytherapy compared with SBRT, but with lower conformity (CI) and heterogeneity (HI) indices in the brachytherapy group [20]; $\mathrm{V}_{100}$ and $\mathrm{D}_{90}$ (BED Gy) prescription doses were nearly identical in both treatments $(91.6 \%$ and $92.4 \% ; 104.7 \mathrm{~Gy}$ and $107.6 \mathrm{~Gy}$, respectively), but $\mathrm{V}_{150}$ was substantially higher with brachytherapy $(64.7 \%$ and $0 \%)$ [20].

A clinical investigation was conducted in China to compare the clinical efficacy and complications of ${ }^{125} \mathrm{I}$ seed brachytherapy and SBRT for recurrent pulmonary metastases from colorectal carcinoma. In the seed implant group, the implantation therapy was well-tolerated by all. In the SBRT group, all patients received only two sessions due to radiation induced lung injury, resulting in only supportive care. Thus, the survival rate was improved in the iodine seed group than the SBRT group at two years [21].

Huo et al. investigated the role of CT-guided ${ }^{125}$ I seed implantation in combination with chemotherapy for the treatment of stage III NSCLC. Brachytherapy in combination with chemotherapy is a viable treatment option for the treatment of NSCLC, and it is important to note that this study found that with this combination therapy, hemoglobin levels before treatment, post-operative $D_{100}$, and the maximum tumor diameter might be major prognostic factors for survival [22].

A publication on brachytherapy for the treatment of lung cancer provides an extensive summary of the indications for brachytherapy for the treatment of lung cancer, treatment options, the results, and adverse events [23]. Detailed review of treatment of both operable and inoperable tumors are discussed. Brachytherapy is a feasible treatment option for radical treatments and for palliative treatment. The use of ${ }^{103} \mathrm{Pd}$ allows for decreased exposure to surrounding tissue and to staff. Furthermore, brachytherapy using permanent implants placed percu- 
taneously are more common in the USA and Japan [23]. The main limitation of the present study is there are alternative treatment methods established that are compared to a very small sample size in this paper. The authors understand that hundreds of patients have been treated in prospective and partly randomized controlled trials using other treatment methods. With increased data showing minimal adverse events and favorable local control, we believe permanent seed implant brachytherapy will become more mainstream throughout the world.

\section{Conclusions}

At an average of 58.1 months follow-up (range, 15 to 145 months), none of the 8 implanted lesions have recurred following original or salvage implant (Figure 1),
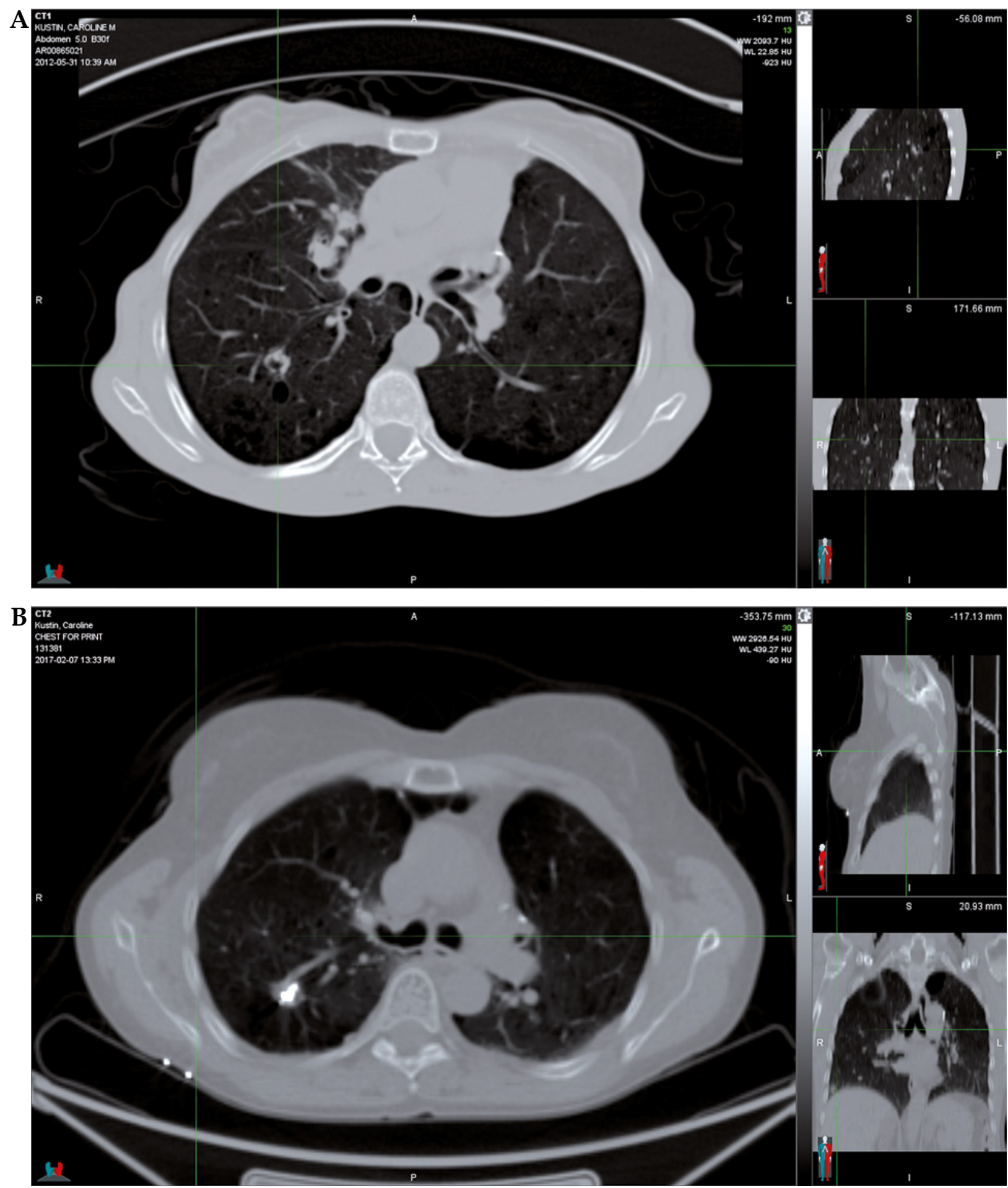

Fig. 1. A) Patient 6: Day of implant. B) Patient 6, 57-month: follow-up 
and there have been no toxicities after seeding. There were no acute complications requiring intervention and no long-term complications. One lesion recurred and required a second implant 10 months after initial implant. The original $\mathrm{D}_{90}$ on this lesion was only $90 \%$. Salvage implant at this site resulted in a D90\% of $273 \%$ and was NED at 43-month follow-up.

In this paper, we have presented a novel outpatient approach for treatment of primary and recurrent lung cancer that is also cost-effective in our practice. The technique is rapid and is not as costly and resource intensive as SBRT, IMRT, proton beam, or RFA. Patients deemed medically inoperable remain candidates for percutaneous CT fluoroscopy-guided permanent seed brachytherapy.

\section{Disclosure}

Authors report no conflict of interest.

\section{References}

1. Curran WJ, Paulus R, Langer CJ et al. Sequential vs. concurrent chemoradiation for stage III non-small-cell lung cancer: randomized phase III trial RTOG 9410. J Natl Cancer Inst 2011; 103: 1452-1460.

2. Siegel R, Naishadham D, Jemal A. Cancer statistics. Cancer J Clin 2013; 63: 11-30.

3. Rajpara RS, Schreibmann E, Fox T et al. Locoregional tumor failure after definitive radiation for patients with stage III non-small cell lung cancer. Radiat Oncol 2014; 9: 187.

4. Molina JR, Yang P, Cassivi SD et al. Non-small cell lung cancer: epidemiology, risk factors, treatment, and survivorship. Mayo Clin Proc 2008; 83: 584-594.

5. Yashiro H, Nakatsuka S, Inoue M et al. Factors affecting local progression after percutaneous cryoablation of lung tumors. J Vasc Interv Radiol 2013; 24: 813-821.

6. Doggett SW, Chino S, Stevens G. CT guided outpatient percutaneous radioisotope implantation of chest tumors. Radiother Oncol 1996; 39: S1.

7. Doggett SW. CT guided radioisotope seed implantation for chest tumors. GEC/ESTRO ABS, 15 May 1996; Tours, France.

8. Doggett SW, Chino S, Lempert T. Management of adenoid cystic carcinoma metastatic to lung with CT fluoroscopic guided brachytherapy. American Brachytherapy Society Annual Meeting, 2014 April 3-5, San Diego, CA, USA.

9. Doggett SW, Chino S, Lempert T et al. Percutaneous CT-fluoroscopic guided radioisotope seed placement for the management of adenoid cystic carcinoma of the trachea. Brachytherapy 2017; 16: 639-645.

10. Michalski J, Mutic S, Eichling J et. al. Radiation exposure to family and household members after prostate brachytherapy. Int J Radiat Oncol Biol Phys 2003; 56: 764-768.

11. Ozaki M. Development of a real time reconstruction system for CT fluorography. Med Rev 1995; 53: 12-17.

12. Aupérin A, Le Pechoux C, Rolland E et al. Meta-analysis of concomitant versus sequential radiochemotherapy in locally advanced non-small-cell lung cancer. J Clin Oncol 2010; 28: 2181-2190.

13. Bradley JD, Paulus R, Komaki R et al. Standard-dose versus high-dose conformal radiotherapy with concurrent and consolidation carboplatin plus paclitaxel with or without cetuximab for patients with stage IIIA or IIIB non-small-cell lung cancer (RTOG 0617): a randomised, two-by-two factorial phase 3 study. Lancet Oncol 2015; 16: 187-199.

14. Auliac JB, Chouaid C, Greillier L et al. Randomized open-label non-comparative multicenter phase II trial of sequential erlotinib and docetaxel versus docetaxel alone in patients with non-small-cell lung cancer after failure of first-line chemotherapy: GFPC 10.02 study. Lung Cancer 2014; 85: 415-419.

15. Park S, Keam B, Kim SH et al. Pemetrexed singlet versus non-pemetrexed-based platinum doublet as second-line chemotherapy after first-line epidermal growth factor receptor (EGFR) tyrosine kinase inhibitor failure in non-small cell lung cancer patients with EGFR mutations. Cancer Res Treat 2015; 47: 630-637.

16. Pao W, Miller VA, Politi KA et al. Acquired resistance of lung adenocarcinomas to gefitinib or erlotinib is associated with a second mutation in the EGFR kinase domain. PLoS Med 2005; 2: e73.

17. Wong MK, Lo AI, Lam B et al. Erlotinib as salvage treatment after failure to first-line gefitinib in non-small cell lung cancer. Cancer Chemother Pharmacol 2010; 65: 1023-1028.

18. Lin JJ, Cardarella S, Lydon CA et al. Five-year survival in EGFR-mutant metastatic lung adenocarcinoma treated with EGFR-TKIs. I Thorac Oncol 2015; 11: 556-565.

19. Keating GM. Nivolumab: A review in advanced squamous non-small cell lung cancer. Drugs 2015; 75: 1925-1934.

20. Li R, Zhang Y, Yuan $Y$ et al. Dosimetric comparison of CT-guided iodine-125 seed stereotactic brachytherapy and stereotactic body radiation therapy in the treatment of NSCLC. PLoS One 2017; 12: e0187390.

21. Li J, Zhang L, Xie Q et al. Comparison of clinical efficacy and complications of 125-I seeds brachytherapy and stereotactic body radiation therapy for recurrent pulmonary metastases from colorectal carcinoma. J Contemp Brachytherapy 2017; 10: 360-367.

22. Huo $X$, Huo B, Wang $H$ et al. Implantation of computed tomography-guided Iodine- 125 seeds in combination with chemotherapy for the treatment of stage III non-small cell lung cancer. I Contemp Brachytherapy 2017; 9: 527-534.

23. Skowronek J. Brachytherapy in the treatment of lung cancer a valuable solution. J Contemp Brachytherapy 2015; 7: 297-311. 